\title{
Spatially resolved variations of the IMF mass normalization in early-type galaxies as probed by molecular gas kinematics
}

\author{
Timothy A. Davis ${ }^{1 \star}$ and Richard M. McDermid ${ }^{2,3}$ \\ ${ }^{1}$ School of Physics and Astronomy, Cardiff University, Queens Buildings, The Parade, Cardiff CF24 3AA, UK \\ ${ }^{2}$ Department of Physics and Astronomy, Macquarie University, Sydney, NSW 2109, Australia \\ ${ }^{3}$ Australian Astronomical Observatory, PO Box 915, Sydney, NSW 1670, Australia
}

Accepted 2016 September 15. Received 2016 September 12; in original form 2016 May 5

\begin{abstract}
We here present the first spatially resolved study of the initial mass function (IMF) in external galaxies derived using a dynamical tracer of the mass-to-light ratio $(\mathrm{M} / \mathrm{L})$. We use the kinematics of relaxed molecular gas discs in seven early-type galaxies (ETGs) selected from the $\mathrm{ATLAS}^{3 \mathrm{D}}$ survey to dynamically determine $\mathrm{M} / \mathrm{L}$ gradients. These $\mathrm{M} / \mathrm{L}$ gradients are not very strong in the inner parts of these objects, and galaxies that do show variations are those with the highest specific star formation rates. Stellar population parameters derived from star formation histories are then used in order to estimate the stellar IMF mismatch parameter, and shed light on its variation within ETGs. Some of our target objects require a light IMF, otherwise their stellar population masses would be greater than their dynamical masses. In contrast, other systems seem to require heavier IMFs to explain their gas kinematics. Our analysis again confirms that IMF variation seems to be occurring within massive ETGs. We find good agreement between our IMF normalizations derived using molecular gas kinematics and those derived using other techniques. Despite this, we do not see find any correlation between the IMF normalization and galaxy dynamical properties or stellar population parameters, either locally or globally. In the future, larger studies which use molecules as tracers of galaxy dynamics can be used to help us disentangle the root cause of IMF variation.
\end{abstract}

Key words: ISM: molecules-galaxies: elliptical and lenticular, $\mathrm{cD}$-galaxies: evolutiongalaxies: ISM - galaxies: kinematics and dynamics.

\section{INTRODUCTION}

The stellar initial mass function (IMF) is one of the most fundamental, and hotly debated, observational topics in astrophysics. Observations of stars within our own Milky Way suggest that the gravitational collapse of molecular clouds leads to star formation and the birth of a population of stars whose masses can be well described by a single mass function (Salpeter 1955; Kroupa 2001; Chabrier 2003). This mass function appears to be universal across the range of environments which we are able to probe within our own Galaxy (Kroupa 2002; Bastian, Covey \& Meyer 2010).

In the early Universe, and in other extragalactic environments, however, conditions can be very different than those found locally. Understanding if the IMF is universal in these places, or if it varies (and why) is crucial to allow interpretations of observations, impacting almost all areas of astrophysics. For instance, calibrations that allow estimation of star formation rates, stellar mass-loss return rates and even total stellar masses rely intimately on an assumed IMF (e.g. Clauwens, Schaye \& Franx 2016).

Recently, evidence for the non-universality of the stellar IMF of the most massive early-type galaxies (ETGs) has begun to mount. This evidence comes from three independent techniques which can probe the IMF in the unresolved stellar populations of ETGs, based on the modelling of stellar kinematics (e.g. Cappellari et al. 2012; Dutton et al. 2013; Tortora, Romanowsky \& Napolitano 2013); utilizing strong gravitational lensing (e.g. Auger et al. 2010; Treu et al. 2010); or via gravity-sensitive spectroscopic features in galaxy spectra (e.g. Cenarro et al. 2003; van Dokkum \& Conroy 2010; Conroy \& van Dokkum 2012; Ferreras et al. 2013).

Stellar kinematic and lensing studies are sensitive to the stellar mass-to-light ratio (M/L) and the major uncertainty in such studies relates to the contribution of the dark matter halo to the potential of galaxies. Spectroscopic constraints are mostly sensitive to the ratio between giant and dwarf stars and its primary uncertainty is the degeneracy between the IMF parameters and those of the underlying stellar populations, most notably the effect of variations in the individual elemental abundances. 
Table 1. Properties of the ETGs included in this study.

\begin{tabular}{|c|c|c|c|c|c|c|c|}
\hline $\begin{array}{l}\text { Name } \\
\text { (1) }\end{array}$ & $\begin{array}{l}\text { Distance } \\
(\mathrm{Mpc}) \\
(2)\end{array}$ & $\begin{array}{c}M_{K s} \\
(\mathrm{mag}) \\
(3)\end{array}$ & $\begin{array}{c}\sigma_{e} \\
\left(\mathrm{~km} \mathrm{~s}^{-1}\right) \\
(4)\end{array}$ & $\begin{array}{c}R_{e} \\
(\mathrm{kpc}) \\
(5)\end{array}$ & $\begin{array}{c}R_{\max } / R_{e} \\
\text { (6) }\end{array}$ & $\begin{array}{c}\log _{10}\left(M_{\text {gas }} / M_{*}\right) \\
\text { (7) }\end{array}$ & $\begin{array}{c}\alpha_{\text {dyn }}(\mathrm{C}+12) \\
(8)\end{array}$ \\
\hline NGC0524 & 23.3 & -24.71 & 220 & 4.9 & 0.49 & -3.43 & 0.60 \\
\hline NGC3607 & 22.2 & -24.74 & 206 & 4.1 & 0.21 & -2.84 & 0.72 \\
\hline NGC3665 & 33.1 & -24.92 & 216 & 5.0 & 0.51 & -2.44 & 0.96 \\
\hline NGC4429 & 16.5 & -24.32 & 177 & 3.3 & 0.52 & -2.78 & 0.92 \\
\hline NGC4459 & 16.1 & -23.89 & 158 & 2.8 & 0.13 & -2.67 & 0.70 \\
\hline NGC4526 & 16.4 & -24.62 & 208 & 3.5 & 0.35 & -2.65 & 0.94 \\
\hline IC0719 & 29.4 & -22.70 & 128 & 1.8 & 1.11 & -2.29 & $* 2.06$ \\
\hline
\end{tabular}

Notes: Column 1 lists the name of each source. Column 25 are the distance, $K s$-band absolute magnitude, velocity dispersion within one effective radius and effective radius of each object. These are reproduced from Cappellari et al. (2011,2013). Column 6 contains the ratio of $R_{\max }$ (the radius at which the rotation profile becomes flat; these figures taken from Davis et al. 2014) to the effective radius $R_{e}$. Column 7 lists the gas fraction (molecular plus atomic) within the inner regions of these objects, as described in Davis et al. (2014). The stellar mass used here is the dynamical mass derived from jeans modelling in Cappellari et al. (2013). Column 8 contains the $\alpha_{\text {dyn }}$ value derived by Cappellari et al. (2012). A star denotes values of $\alpha_{\text {dyn }}$ considered unreliable by Cappellari et al. (2012) due to the presence of strong population gradients.

Studies utilizing these techniques seem to agree that a variation in the IMF is occurring, with more massive ETGs having heavier IMFs. These studies disagree, however, on what the primary driving mechanism for such a variation is, with some studies favouring galaxy velocity dispersion (e.g. Cappellari et al. 2012; La Barbera et al. 2013; Posacki et al. 2015), others' metallicity (e.g. MartínNavarro et al. 2015b) or (alpha-)element abundances (e.g. Conroy $\&$ van Dokkum 2012). Some authors have suggested that these studies lack internal consistency, with different analyses of the same objects finding different IMF slopes (Smith 2014). The water has muddied further with the discovery that dwarf elliptical galaxies also seem to have non-universal IMFs, spanning a similar range of giant galaxies, while having vastly different properties (Tortora, La Barbera \& Napolitano 2016).

In this work, we introduce a new complementary technique to probe the IMF in galaxies. We use the kinematics of the cold molecular gas reservoirs in massive ETGs to constrain their mass profiles. By combining these profiles with observations of the galaxies' stellar luminosity profile (and stellar population parameters), we are able to derive $\mathrm{M} / \mathrm{Ls}$ and constrain the IMF in a radially resolved manner. Studies of the radial variation of the IMF within individual objects are relatively new (see e.g. La Barbera et al. 2016; Zieleniewski et al. 2015), but have significant diagnostic power to determine the astrophysics behind IMF variation. For instance, Martín-Navarro et al. (2015a) find significant IMF gradients in two massive ETGs, while a lower mass object showed little variation. If confirmed, this could imply that the enhanced fraction of low-mass stars causing IMF variation is only present in galaxy bulges which formed violently at high redshift.

In Section 2 of this paper, we present details of our sample selection and the properties of the target objects. Section 3 details the observation parameters and reduction for the new data used in this work. In Section 4, we present details of the method we use to constrain the IMF. We then present our results in Section 5 and discuss them in Section 6, before concluding in Section 7.

\section{SAMPLE}

In this work, we require resolved maps of the molecular interstellar medium (ISM) in massive ETGs. We thus selected objects from the ATLAS $^{3 \mathrm{D}}$ survey (Cappellari et al. 2011), which has provided the largest sample of interferometrically mapped ETGs to date (Alatalo et al. 2013). In addition, by selecting from this survey, we have access to all the required ancillary data (including integral field spectroscopy and optical imaging) required to complete our analysis. We select ETGs from the ATLAS ${ }^{3 \mathrm{D}}$ survey which have been shown in Alatalo et al. (2013) and Davis et al. (2013a) to have regular, relaxed molecular gas distributions that rotate in the disc plane of their host (Davis et al. 2011b). We require that the molecular discs in these objects are well resolved, with at least three beams radially along their major axis. This leads to a sample of seven objects that we study in this work. These objects span a range of galaxy parameters, with velocity dispersions between 150 and $260 \mathrm{~km} \mathrm{~s}^{-1}$ (Cappellari et al. 2013), stellar metallicities of a 2/3-1.5 times solar and mean mass-weighted stellar ages of 7-14 Gyr (McDermid et al. 2015). Other salient properties of these objects are described in Table 1.

For three of these objects, we utilize the $\mathrm{CO}(1-0)$ interferometric data of Alatalo et al. (2013), taken as part of the ATLAS ${ }^{3 \mathrm{D}}$ Combined Array for Research in Millimetre-wave Astronomy (CARMA) survey. For NGC0524, we use the PdBI observations of $\mathrm{CO}(1-0)$ from Crocker et al. (2011). For NGC3665 and NGC4526, we utilize the higher resolution $\mathrm{CO}(2-1)$ observations of Onishi et al., in preparation and Davis et al. (2013b), respectively. We refer readers to these papers for a full description of the data acquisition and reduction. In this analysis, we utilize the full, cleaned $\mathrm{CO}$ data cubes created by the above authors. For NGC4459, we make use of new high-resolution CARMA $\mathrm{CO}(2-1)$ data and we describe these observations in Section 3 below.

\section{CARMA OBSERVATIONS OF NGC4459}

In this study, we utilize new high-resolution CARMA data for the fast-rotating ETG NGC4459. ${ }^{12} \mathrm{CO}(2-1)$ observations of this object were taken in the B, C and D configurations of CARMA between 2010 November 25 and 2011 May 3. The combination of these arrays means that we are sensitive to emission on scales of 0.3 24 arcsec at the frequency of $\mathrm{CO}(2-1)$. Three $250 \mathrm{MHz}$ correlator windows were placed over the $\mathrm{CO}(2-1)$ line, giving a continuous velocity coverage of $>800 \mathrm{~km} \mathrm{~s}^{-1}$ with a raw velocity resolution of $\approx 4.1 \mathrm{~km} \mathrm{~s}^{-1}$. This is sufficient bandwidth and channel resolution to properly cover and sample the line. 


\subsection{Data reduction}

The raw CARMA visibility data were reduced using standard procedures, as documented in Alatalo et al. (2013). We briefly summarize the salient details here. The Multichannel Image Reconstruction Image Analysis and Display (MIRIAD) package (Sault, Teuben \& Wright 1995) was used the reduce the data. For each track, the raw data were Hanning-smoothed in velocity and baselines that show decorrelation on calibrator sources were flagged. The data were then line-length corrected and the bandpass was calibrated using observations of bright quasar 3C273. The atmospheric phase offsets present in the data were determined using 3C273 as a phase calibrator, observed at regular $(\approx 15 \mathrm{~min})$ intervals. Amplitude calibration was performed using Mars. Flux calibration uncertainties are assumed to be up to 20 per cent. After the data were satisfactorily processed, the gain solutions derived from the nearby calibrator were applied to the source.

We then used MIRIAD to combine and image the resultant visibility files for each track, producing a $3 \mathrm{D}$ data cube. The $u v$-data sets were transformed into RA-Dec.-velocity space (with velocities determined with respect to the rest frequency of the $\mathrm{CO}(2-1)$ line). We here use data with a channel width of $10 \mathrm{~km} \mathrm{~s}^{-1}$. Pixels of 0.2 arcsec $\times 0.2$ arcsec were chosen as a compromise between spatial sampling and resolution, typically giving approximately 5 pixels across the beam major axis. One arcsecond corresponds to a physical scale of $\approx 78 \mathrm{pc}$ in this source. We imaged the area within the primary beam of the $10 \mathrm{~m}$ antennas $(\approx 54 \mathrm{arcsec})$. The MIRIAD imaging task INVERT was run with the mosaicking option to properly scale the data and account for the different primary beamwidths.

The data presented here were produced using natural weighting, yielding a synthesized beam of 1.08 arcsec $\times 0.98$ arcsec (a linear resolution of $\approx 84 \times 76 \mathrm{pc}$ ). The dirty cubes were cleaned in regions of source emission to a threshold equal to the root mean square (rms) of the dirty channels. The clean components were then added back and re-convolved using a Gaussian beam of full-width at halfmaximum (FWHM) equal to that of the dirty beam. This produced the final, reduced and fully calibrated data cube for NGC4459, which has an rms noise level of $4.21 \mathrm{mJy}^{\text {beam }^{-1}}$.

\subsection{Data products}

The clean fully calibrated data cube was used to create moment maps: a zeroth moment (or integrated intensity) map and a first moment (mean velocity) map. In order to create these, the clean data cube was Hanning-smoothed in velocity and Gaussian-smoothed spatially (with an FWHM equal to that of the beam) and masks were created by selecting all pixels above a fixed flux threshold of $2.5 \sigma$, adjusted to recover as much flux as possible in the moment maps while minimizing the noise. The moment maps were then created using the unsmoothed cubes within the masked regions only. In addition, the major-axis position-velocity diagram (taken with a PA of $269^{\circ}$, as determined in Davis et al. 2011b) and total spectrum were extracted. These data products are shown in Fig. 1.

\subsection{Gas morphology}

Fig. 1 shows the morphology of the gas in NGC4459. We detect gas in an oval region, coincident with the dust visible in Hubble Space Telescope (HST) imaging. The dust is distributed in a flocculent disc, with a sharp outer edge at a semimajor axis of $8.5 \operatorname{arcsec}$ and only a few faint spiral dust features exist beyond this. The $\mathrm{CO}$ in this object also appears to be truncated at the outer edge of the dust disc, although the faint spiral features in the north-west of the galaxy do have some detected emission. The inclination of $47^{\circ}$ estimated by previous studies (e.g. Young, Bureau \& Cappellari 2008; Davis et al. 2011a) would be fully consistent with the gas being distributed in a flat, circular disc in the galaxy plane. Kinematic evidence also suggests that the gas in this object is distributed in a regularly rotating disc. The 'spider diagram' seen in the channel maps is regular and symmetric and (as our later analysis will show) can be used to predict an identical inclination to that estimated from photometry. The molecular gas disc is flocculent, with various gas peaks that likely relate to marginally resolved giant molecular clouds within the disc. Unlike in lenticular galaxy NGC4526 (Davis et al. 2013b; Utomo et al. 2015b), however, some molecular gas emission is present at all positions in-between these peaks, suggesting the presence of a large population of unresolved clouds.

In the HST image, one can see that from a radius of about 3.75 arc$\mathrm{sec}$, two dusty spiral arms connect the main disc with an inner dust ring of semimajor axis $\approx 2.6$ arcsec. Young et al. (2008) conclude that these features are trailing spirals. The arm features do not seem to be associated with strong peaks in the gas emission, however, they do seem to affect the gas within the dust ring. Three peaks of emission are detected within the inner ring, which seem to form a gas ring when connected, with outer radius traced by the dust structure. Two of these peaks have approximately similar strength and lie upstream of the point where the spiral dust structures connect to the ring. These peaks could be caused by buildup of material, as gas rotating around the ring meets material inflowing along the spiral structures. This hypothesis is supported by the line-of-sight CO spectra extracted from the position of these peaks, which have wings towards low velocity (i.e. towards the galaxy systemic) which cannot be reproduced by our kinematic modelling assuming circular orbits. The other peak to the south of the inner ring is less bright and may be a single giant molecular cloud/association flowing around the ring.

\subsection{Comparison with existing observations}

Combes et al. (2007) observed CO(1-0) and CO(2-1) in NGC4459 with the IRAM-30 $\mathrm{m}$ telescope, finding a total $\mathrm{CO}(2-1)$ flux of 86.5 $\pm 3.1 \mathrm{Jy} \mathrm{km} \mathrm{s}^{-1}$. We compare the total spectrum extracted from our observations to the $\mathrm{CO}(2-1)$ data of these authors in Fig. 1, finding that the total flux is $\approx 40$ per cent higher in our interferometric data. This likely arises because the beam size of the IRAM-30 $\mathrm{m}$ at $230 \mathrm{GHz}$ is 11 arcsec, similar to the total size of the molecular gas disc. Making mock observations of our resolved data cube using a Gaussian beam shape of this size yields an almost identical spectrum to that in Combes et al. (2007). We are hence confident that we have not resolved out significant flux in the central parts of this object.

Resolved observations of the $\mathrm{CO}(1-0)$ line in this object were presented in Young et al. (2008), with a beamsize of 9.0 arcsec $\times 5.5$ arcsec. Our observations are entirely consistent with those of Young et al. (2008). Convolving our observations with an asymmetric beam to match those in Young et al. (2008) yields very similar moment maps (and a matching major-axis position-velocity diagram). We thus conclude that that $\mathrm{CO}(1-0)$ and $\mathrm{CO}(2-1)$ transitions arise from gas which is entirely co-spatial in this object, with identical kinematics. This is expected, given the low-excitation temperatures of these transitions (which means that they should trace the bulk of the molecular material in high-metallicity objects like NGC4459). 

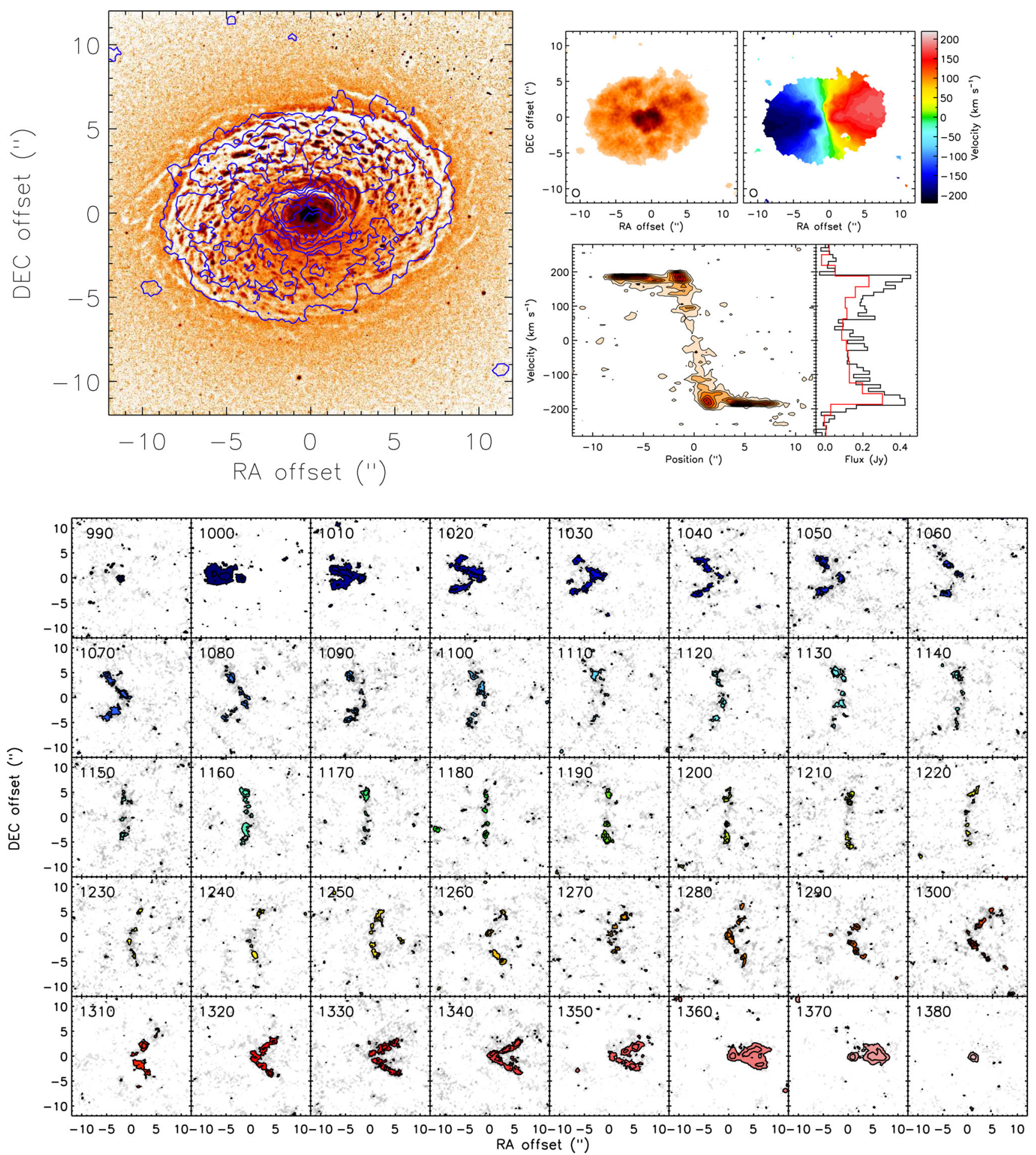

Figure 1. New CARMA observations of NGC4459. Top left: Hubble Space Telescope UVIS F475W image of the galaxy centre, which has been unsharpmasked to show the dust disc. Overlaid are the contours of the $\mathrm{CO}(2-1)$ integrated intensity map. Top right, upper panel: zeroth and first moment (integrated intensity and velocity) maps of the $\mathrm{CO}(2-1)$ emission. Top right, lower panel: position-velocity diagram of the $\mathrm{CO}$ emission extracted along the kinematic major axis. Also shown in the right-hand panel is the total CO(2-1) spectrum of NGC4459 (black line). Overlaid on this in red is the IRAM-30m observation of Combes, Young \& Bureau (2007). Bottom: grey scale shows the channel maps in the velocity interval where emission is detected (990-1380 km s $\left.{ }^{-1}\right)$. The coloured region with black contours shows the areas detected with above a $3 \sigma$ significance.

\section{METHOD}

In this work, we aim to constrain the mass profile of our target ETGs, and thus their $\mathrm{M} / \mathrm{L}$ gradients. In order to do this, we model the rotation of the molecular gas. We also require an estimate of the stellar population properties and how these vary with radius. In this section, we explain the methods used to do this, dealing with molecular gas kinematic modelling in Section 4.1 and stellar 
population analyses in Section 4.2. Combining these two elements allows us to estimate the IMF mismatch parameter (Section 4.3) and how it varies radially with respect to local galaxy properties (Section 5).

\subsection{Molecular gas kinematic modelling}

In order to estimate the $\mathrm{M} / \mathrm{L}$ as a function of radius in our earlytype sources, we used a forward modelling approach. We utilized the KINematic Molecular Simulation (KinMS ${ }^{1}$ ) mm-wave observation simulation tool of Davis et al. (2013a). This tool allows us to input guesses for the true gas distribution and kinematics and (taking into account the observational effects of beam-smearing, disc thickness, velocity dispersion, binning, etc) produce a simulated data cube which can be compared with the observed data.

In order to determine the rotation speed of the gas as a function of radius, as in Davis (2014), we used the Markov Chain Monte Carlo (MCMC) code KINMS_MCMC that couples to the KinMS routines and allows us to fit the data and obtain the full bayesian posterior probability distribution for the fitted parameters. This code fits the entire data cube produced by the interferometer, rather than simply the position-velocity diagram (as was done in Davis et al. 2013b). The simulations used a beam, pixel size and velocity resolution identical to our observations.

\subsubsection{Gas distribution and kinematics}

For each object, we created a simple model of the gas distribution which is then used as an input to the modelling code. We do not aim to reproduce all the internal features of gas distribution, only the broader details of at which locations gas is present or absent. In NGC0524 and NGC3607, we chose to distribute the gas in an exponential disc. This simple, zeroth-order form (which has been shown to be appropriate in most ETGs; Davis et al. 2013a) provides a good match to the observed morphology of the gas in these objects. In NGC3665 and NGC4429, we used exponential discs with an inner cutoff (as explained in Onishi et al., in preparation). In NGC4459, we again used an exponential disc model with an inner cutoff, with an additional Gaussian enhancement of the gas density at the radius of the molecular ring seen in our observations (see Section 3). In NGC4525, we use a three-ring model, as previously presented in Davis (2014). We note, however, that (as explored in detail in Davis 2014) using a more simple form for the gas distribution does not bias our results. The exponential disc and ring radii (and cutoff radii) were all left as free parameters, which were modified by the KINMS_MCMC code to obtain a good fit.

In addition to the free parameters related to the gas distribution, various other free parameters of the gas disc are included in the model. These are the total flux, position angle and inclination of the gas disc, as well as its kinematic centre (in RA and Dec.) and its systemic velocity. We find no evidence of disc warps in the sample galaxies, so the inclination and position angle are fitted as a single value, which applies in each radial bin.

We assume implicitly that the gas in in circular rotation within the potential and hence, the gas velocity varies only radially. We parametrize the kinematics in radial annuli, of one beamwidth across. As our sources have various physical extents and our observations have different beamsizes, we are able to fit between 3 and 10 independent radial bins per source. In addition to the free parameters

\footnotetext{
${ }^{1}$ available at https://github.com/TimothyADavis/KinMS
}

controlling the gas rotation curve, we also include a free parameter for the internal velocity dispersion of the gas disc, which is assumed to be constant radially within our objects. For those galaxies with high-resolution data (NGC0524, NGC3665, NGC4526), we include the gravitational effect of the measured Supermassive Black Hole in our model, with its mass fixed to the value found by other authors (Krajnovic et al. 2009; Onishi et al., in preparation; Davis et al. 2013b). We then allow the MCMC code to estimate the circular velocities within each of our bins (see Section 4.1.2 for more details).

In order to convert the derived velocity profiles to dynamical M/L measurements, we here parametrize the luminous matter distribution using multi-Gaussian expansion (MGE; Emsellem, Monnet \& Bacon 1994) models of the stellar light distribution. These were constructed from HST images (at the longest wavelength available, in order to minimize dust contamination), and from $r$-band Sloan Digital Sky Survey (SDSS) images in cases where this was not possible. In IC719, we use Spitzer Space Telescope $3.6 \mu \mathrm{m}$ observations, as the source is very dusty. Assuming the Gaussian density distribution assumption made when constructing an MGE holds, this model of the stellar light can easily be de-projected using the observed inclination (as fitted by KINMS_MCMC). It then directly predicts the circular velocity of the gas caused by the luminous matter, modulo the stellar M/L.

At each step of the MCMC chain, we use the MGE to predict the rotation curve of the galaxy at some inclination, imposing an M/L of unity. Then, using the basic relationship

$V=\sqrt{\frac{G M}{r}}=\sqrt{\frac{G\left(L \frac{M}{L}\right)}{r}}=\sqrt{\frac{G L}{r}} \sqrt{\frac{M}{L}}$,

we can multiply the derived rotation curve by the $\mathrm{M} / \mathrm{L}$ factor chosen at this step for each radial bin. We define this value at the bin centre radius and interpolate linearly between bins. Using stepwise transitions or higher order splines to interpolate between points does not change our results. Note that although equation (1) is only formally valid for a spherical mass distribution, the proportionality is valid generally.

In this way, comparing the derived rotation curve with that expected from the luminous matter alone allows us to estimate the dynamical $\mathrm{M} / \mathrm{L}$ at each radius, which can then be compared with that predicted from stellar population analyses.

\subsubsection{Fitting process}

In order to ensure our kinematic fitting process converges, we set reasonable priors on some of the parameters. The kinematic centre of the galaxy was constrained to lie within one beamwidth of the optical galaxy centre position (but good fits are always found well within this). The systemic velocity was allowed to vary by $\pm 50 \mathrm{~km}$ $\mathrm{s}^{-1}$ from that found by optical analyses (but again good fits are always found in the inner part of this range). The gas velocity dispersion was constrained to be less than $50 \mathrm{~km} \mathrm{~s}^{-1}$ (but is always found to be low, $<12 \mathrm{~km} \mathrm{~s}^{-1}$ ), and the disc scalelength/cutoffs constrained to be less than 20 arcsec. The M/L prior in each radial bin allows variation between 0.1 and 20 . The inclination of the gas disc is allowed to vary over the full physical range allowed by the MGE model. A flat prior was used on each of these parameters (an assumption of maximal ignorance).

Once the MCMC chains were converged, we ran the final iteration 30000 times (with a 10 per cent burn-in) to produce our final posterior probability distribution. These probability surfaces were 
then marginalized over the other parameters in order to produce an estimate of each parameter and its associated 1 and $3 \sigma$ errors.

\subsection{Stellar population analysis}

Using the publicly available spectral data cubes from the Atlas3D survey, ${ }^{2}$ we derive the spatially-resolved stellar M/L from the integrated stellar light using spectral fitting. Spectra are first binned to a minimum signal-to-noise ratio of 80 , using the Voronoi tessellation method of Cappellari \& Copin (2003). The penalised pixel fitting (PPXF) algorithm of Cappellari \& Emsellem (2004) was then used, together with simple stellar population (SSP) templates taken from the MIUSCAT model library (Vazdekis et al. 2012). Regions of the spectra potentially affected by emission lines (namely $\mathrm{H} \beta$ and [O III]) are excluded from the fit. The selected templates span $0.1-$ $14 \mathrm{Gyr}$ in age and -1.71 to 0.2 in $[\mathrm{Z} / \mathrm{H}]$, giving 264 simultaneously fitted templates. We employ linear regularization constraints as per McDermid et al. (2015) to obtain smooth weight distributions in the $2 \mathrm{D}$ space of age and metallicity. The resulting weights, $w_{i}$, are used to compute the mass-weighted $\mathrm{M} / \mathrm{L}$ as

$M / L_{\mathrm{pop}, \mathrm{salp}}=\frac{\sum w_{i} M_{*+\mathrm{rem}, i}}{\sum w_{i} L_{X, i}}$,

where $M_{*+\mathrm{rem}, i}$ is the mass existing in stars and remnants and $L_{X, i}$ is the luminosity in the given filter passband $X$, both corresponding to the age and metallicity associated with weight $w_{i}$ and assuming a unimodal power-law IMF of the form: $\zeta(m) \propto m^{-2.35}$ (Salpeter 1955). This procedure is fully consistent with that employed in Cappellari et al. (2012).

$\mathrm{M} / \mathrm{Ls}$ for photometric bands falling outside the MIUSCAT spectral range come from the photometric predictions of Vazdekis et al. (1996), available from the MILES website for ages and metallicities consistent with the MIUSCAT models. For overlapping optical bands, these M/L predictions agree with the baseline MIUSCAT models with a standard deviation of 0.04 . For the Spitzer $3.6 \mu \mathrm{m}$ band, we use the MIUSCAT-IR models of Röck et al. (2015). Note that these models only extend down to a metallicity $([\mathrm{Z} / \mathrm{H}])$ of -0.4 . For objects with significant low-metallicity populations, this means that we may somewhat underestimate $3.6 \mu \mathrm{m}$ stellar population M/Ls (see e.g. Meidt et al. 2014).

Random errors on the individual M/L measurements were derived using Monte Carlo simulations of the PPXF spectral fit without regularization applied, and taking the standard deviation of the resulting $\mathrm{M} / \mathrm{L}$ distribution. Typical $\mathrm{M} / \mathrm{L}$ error values derived in this way are around $\pm 0.14 \mathrm{M}_{\odot} / \mathrm{L}_{\odot}$.

To explore correlations of the spatially resolved IMF and stellar population parameters, as has been done in the recent literature (e.g. Conroy \& van Dokkum 2012; McDermid et al. 2014; Martín-Navarro et al. 2015b), we employ line index measurements to derive the SSP-equivalent parameters of age, metallicity and alpha-enhancement. We use a combination of Hbeta, Fe5015 and Mgb Lick index measurements cleaned for ionized gas emission lines using the above PPXF spectral fits and find the best-fitting SSP model from Schiavon (2007) that simultaneously reproduces these indices. A full comparison of how these SSP parameters compare with the mass-weighted parameters derived from spectral fitting is presented for these galaxies (as part of the larger ATLAS ${ }^{3 \mathrm{D}}$ Survey) in McDermid et al. (2015).

\footnotetext{
${ }^{2}$ Available from www.purl.org/atlas $3 \mathrm{~d}$
}

We note that these SSP-equivalent parameters are only used for exploring correlations with our inferred IMF mismatch parameter described below. The $\mathrm{M} / \mathrm{L}_{\text {pop,salp }}$ itself is derived using spectral fitting, which allows us to account for the influence of a spread of ages and metallicities on the integrated light through the combination of fitted templates. Regularization ensures a maximally smooth star formation history that still fits the data within the observational uncertainties, though we find that the resulting $\mathrm{M} / \mathrm{L}$ is not significantly dependent on the degree of regularisation used - the average absolute difference between the regularized and non-regularized $\mathrm{M} / \mathrm{L}$ is 0.16 , which is comparable to the random uncertainties.

\subsection{Derivation of IMF mismatch parameters}

Following Cappellari et al. (2012), we define the IMF mismatch parameter $\left(\alpha_{\text {dyn }}\right)$ as

$\alpha_{\mathrm{dyn}}=\frac{M_{\mathrm{dyn}}}{M_{\mathrm{pop}, \mathrm{salp}}}=\frac{M / L_{\mathrm{dyn}}}{M / L_{\mathrm{pop}, \mathrm{salp}}}$,

where $M_{\text {dyn }}$ is the dynamical mass within a bin estimated from the CO kinematics (which makes no assumption on the IMF) and $M / L_{\text {pop,salp }}$ is the stellar $\mathrm{M} / \mathrm{L}$, estimated from the stellar population analysis assuming a Salpeter (1955) IMF as described in the previous subsection. This ratio is equal to unity if the real IMF in the galaxy follows a Salpeter (1955) form, 0.58 for a Chabrier (2003) IMF, 0.65 for a Kroupa (2001) IMF and 1.55 for a Heavy IMF (as discussed in e.g. Cappellari et al. 2012).

\section{RESULTS}

In this section, we present the best-fitting $\mathrm{M} / \mathrm{L}$ and $\alpha_{\mathrm{dyn}}$ gradients for our seven sample objects. We compare the values derived with those found by other authors who used different techniques. We also explore some to the uncertainties that can affect measurements of this type in Section 5.5.

\section{$5.1 \mathrm{M} / \mathrm{L}$ and IMF gradients}

For each object in our sample, we present two plots to show our derived M/L and IMF gradients. The first displays the observed major-axis position-velocity diagram, overplotted with the position-velocity diagram extracted from our model in an identical way. Although the model was actually fitted on the full interferometric data cube, this figure allows the reader to easily assess by eye the quality of the fits.

The second plot contains two panels. The top panel shows the M/L gradient present within the source, as derived from the molecular gas observations in black. Also shown as grey plus symbols are the stellar population $\mathrm{M} / \mathrm{L}$ values derived from the independent Integral Field Unit (IFU) bins at each radius. The mean value and scatter around this within each bin are shown as the red points with error bars. In the bottom panel, we show the IMF mismatch parameter in each bin (the ratio of the black and the red point from the upper plot). For reference, we show lines which correspond to Chabrier, Kroupa, Salpeter and Heavy IMFs. We discuss the fits obtained for each object individually below and note any specific uncertainties individual to the source.

\subsubsection{NGC0524}

NGC0524 is a high-mass isolated fast-rotating ETG, orientated nearly face-on to our line of sight. It has a disc of molecular gas 

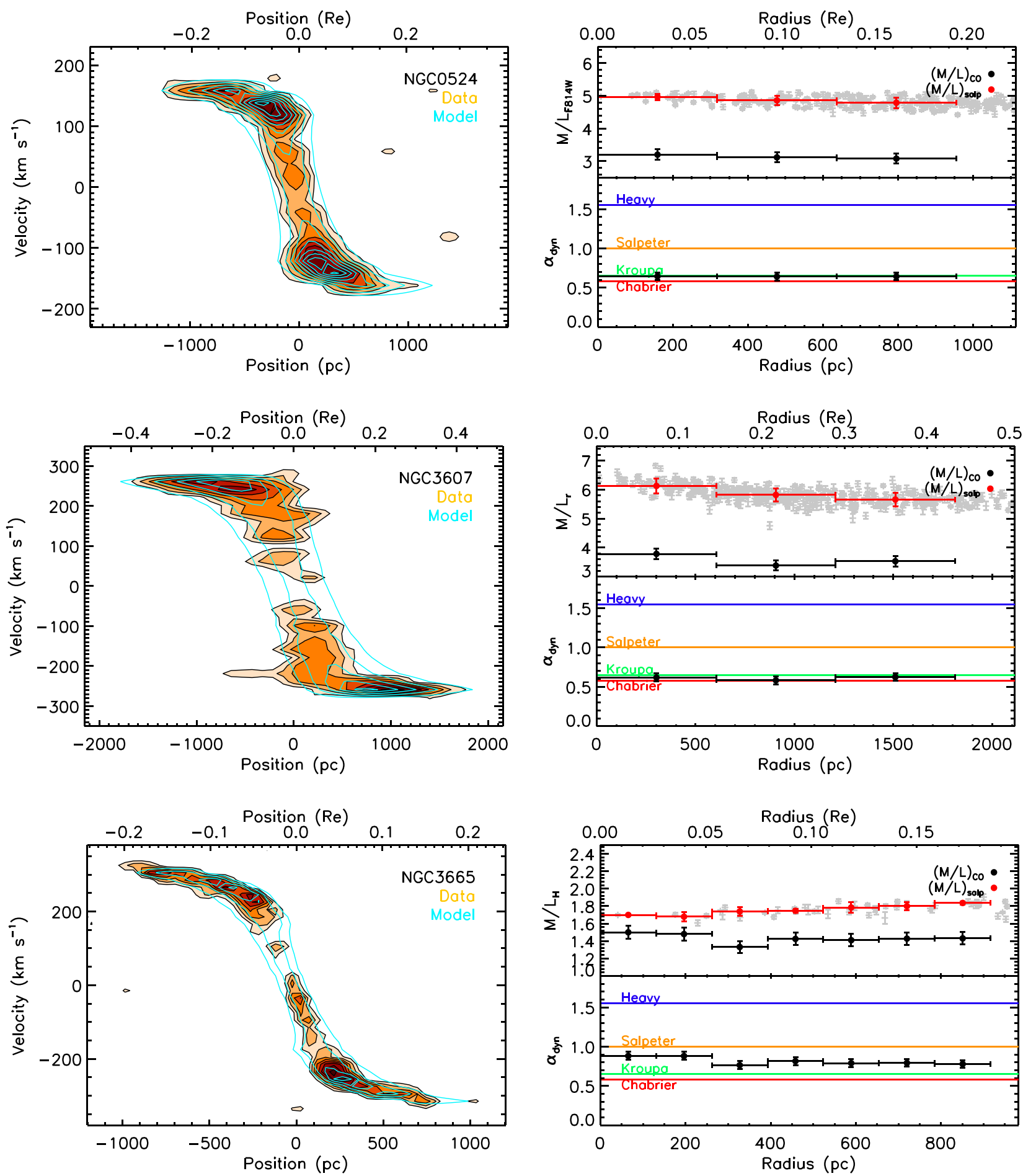

Figure 2. Left: observed major-axis position-velocity diagram for each galaxy in our sample (black contours filled in orange), overplotted in blue with the best-fitting position-velocity diagram extracted from our model (which was actually fitted on the full interferometric data cube) in an identical way. Right: the top panel shows the mass-to-light ratio gradient present within the source, as derived from the molecular gas observations in black. Also shown as grey plus symbols are the stellar population M/L values derived from the independent IFU bins at each radius. The mean value and scatter around this within each bin are shown as the red points with error bars. In the bottom panel, we show the IMF mismatch parameter in each bin (as defined in equation 3). For reference, we show lines which correspond to Chabrier, Kroupa, Salpeter and Heavy IMFs.

and dust that is clearly visible in optical imaging, but very little star formation. The molecular disc is regular and relaxed at our resolution and its surface brightness profile is well fitted with an exponential disc model. We use the mass model from Krajnovic et al. (2009) here, which is constructed using HST F814W observations.
The inclination of this source is low and thus errors in the inclination could introduce large uncertainties. Thanks to the wellresolved $\mathrm{CO}$ disc in this object, however, we can constrain the inclination well and do not expect this to significantly change this result. This is shown graphically in Fig. 2, where all error bars 

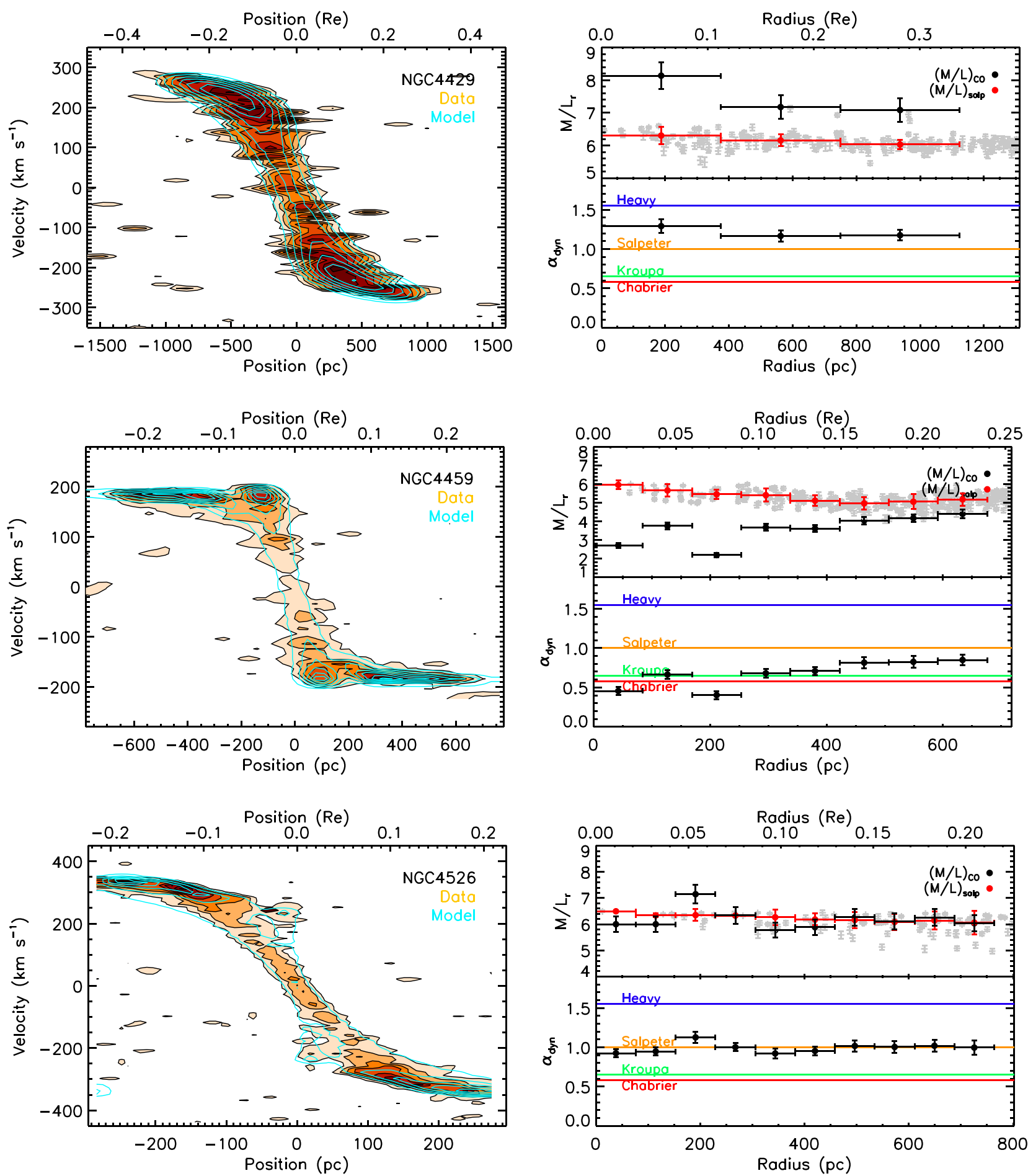

Figure 2 - continued.

on the points have already been marginalized over our inclination uncertainty.

This object has a fairly uniform old stellar population which is predicted to have a high $\mathrm{M} / \mathrm{L}(\approx 5$ in $i$ band, assuming a Salpeter IMF), which increases slightly towards the galaxy centre. However, our $\mathrm{CO}$ observations require a much lower $\mathrm{M} / \mathrm{L}$, which is also modestly centrally peaked. Thus, despite being a massive ETG, the IMF in this object seems to be uniformly light, approximately Kroupa like.

\subsubsection{NGC3607}

NGC3607 is a fast-rotating ETG with a central dust disc that is part of the Leo II Group of galaxies. The molecular disc is regular and relaxed at our resolution, with no sign of the disturbed structures present in the dust distribution. Its surface brightness profile is well fitted with an exponential disc model.

The stellar population $\mathrm{M} / \mathrm{L}$ of this (fast-rotating) elliptical has a mild negative gradient, which is consistent with that predicted 



Figure 2 - continued.

dynamically from our CO observations. The IMF mismatch parameter in this source seems thus to be flat with radius, with a Kroupa/Chabrier type normalization.

\subsubsection{NGC3665}

NGC3665 is an isolated fast-rotating ETG with a strong active galactic nucleus (AGN), which powers large radio jets (Parma et al. 1986; Nyland et al. 2016). The molecular gas and dust in this source is concentrated in a central disc perpendicular to the radio jet. As discussed in Onishi et al., in preparation, the best-fitting surface brightness profile for this object is an exponential disc with a central hole, which we adopt here. We also adopt the mass model used in that study, constructed using $H$-band Near Infrared Camera and Multi-Object Spectrometer observations.

This object has a fairly uniform stellar population. The dynamically inferred $\mathrm{M} / \mathrm{L}$, on the other hand, shows an $\mathrm{M} / \mathrm{L}$ dip at around $\approx 350 \mathrm{pc}$. This is approximately where the $\mathrm{CO}$ distribution peaks in this object so we could be seeing the dilution of the old stellar population with new stars at this location, however, one would also then expect to detect a decrease in the stellar population M/L. Overall, the IMF mismatch parameter seems to lie mid-way between Kroupa and Salpeter in this object.

\subsubsection{NGC4429}

NGC4429 is a lenticular galaxy in the Virgo cluster with a nuclear disc of gas and dust. The molecular disc is regular and relaxed, with a small hole in its centre (radius $\approx 0.45 \mathrm{arcsec}, 36 \mathrm{pc}$; Onishi et al., in preparation). This object has a slight $\mathrm{M} / \mathrm{L}$ gradient over the extent of the molecular gas disc, with an $r$-band $\mathrm{M} / \mathrm{L}$ of $\approx 6$. Our $\mathrm{CO}$ measurements, however, reveal that the central regions of this object contain more mass than expected and the $\mathrm{M} / \mathrm{L}$ varies between $\approx 7.5$ and 6.5 within the inner kiloparsec. This is also reflected in the IMF mismatch parameter, which is marginally consistent with Salpeter in the outer parts but heavier in the galaxy centre.

\subsubsection{NGC4459}

NGC4459 is a lenticular galaxy in the Virgo cluster with a nuclear disc of gas and dust, which is discussed in detail above. Analysis of its stellar population suggests some radial M/L variation, with a gentle rise in the $\mathrm{M} / \mathrm{L}$ with radius and an $\mathrm{M} / \mathrm{L}$ dip around the $200 \mathrm{pc}$ ring/spiral features. This $\mathrm{M} / \mathrm{L}$ feature could arise because the relative amount of recent star formation in this region is higher, as the molecular gas is concentrated at this radius after streaming inwards from the spiral spurs. Our stellar population analysis does detect a younger population in the inner parts of this object, but the low-mass fraction of this component does need lead to a significant change in the predicted M/L. This feature could also be due to the non-circular inflowing motions, as discussed below. Overall, however, despite these features, the IMF appears to be consistent with (or slightly heavier than) Kroupa in this object.

\subsubsection{NGC4526}

NGC4526 is a lenticular galaxy in the Virgo cluster with multiple rings/spirals of molecular gas in its centre (see Davis et al. 2013b; Utomo et al. 2015b). The stellar population M/L of this galaxy is flat with radius within the molecular-rich region, which is consistent with the flat gradient predicted dynamically from our $\mathrm{CO}$ observations. A small peak in the dynamically inferred $\mathrm{M} / \mathrm{L}$ is present around 200pc, consistent with the location of the largest CO ring. Apart from this small feature the IMF mismatch parameter in this source seems to be flat with radius, with a Salpeter-type normalization.

\subsubsection{IC0719}

IC0719 is a relatively low-mass lenticular galaxy $\left(M_{*}=4.3 \times 10^{10}\right.$ $\mathrm{M}_{\odot}$; Cappellari et al. 2013), with strong star formation all across its disc triggered by a recent accretion of molecular gas (Davis et al. 2011b). The gas exactly counter-rotates when compared with the stars and some minor flux asymmetry is still present. Despite this, the gas appears kinematically relaxed and sits in the galaxy midplane, thus it is well suited to our modelling approach. This object is quite young (SSP equivalent age of 3.4 Gyr within $1 \mathrm{Re}$; McDermid et al. 2015), and has a large velocity dispersion gradient, making it an interesting case study for IMF variation studies.

We make use of $3.6 \mu \mathrm{m}$ observations from the S4G survey (Sheth et al. 2010) to derive the luminous stellar mass profile of this galaxy. Non-stellar $3.6 \mu \mathrm{m}$ emission has been subtracted from this image, using the procedure defined in Meidt et al. (2012). Using this band allows us to minimize the effect of dust obscuration, as the source is very dusty even in the SDSS $z$ band and 2MASS near-infrared 
observations are not deep enough. The molecular gas resolution is worse than the Spitzer point spread function at these wavelengths and thus we do not expect the low resolution of the $3.6 \mu \mathrm{m}$ observations to affect our results.

A strong dynamical $\mathrm{M} / \mathrm{L}$ gradient seems to be needed to explain the observed rotation of the gas, as shown in the right-hand panel in Fig. 2. A $3.6 \mu \mathrm{m} \mathrm{M/L} \mathrm{of} \approx 0.2$ is required in the central bin (coloured grey here), while beyond the inner $500 \mathrm{pc}$, the $3.6 \mu \mathrm{m}$ $\mathrm{M} / \mathrm{L}$ becomes $\approx 1.0$. The $\mathrm{M} / \mathrm{L}$ estimated from our stellar population fits at $3.6 \mu \mathrm{m}$ is much more constant, with values around unity. This leads us to predict to a strong $\alpha_{\text {dyn }}$ gradient in this source, with the IMF normalization increasing outwards, but being consistent with Salpeter beyond the innermost 500 pc.

Note that although our line-strength-based metallicity measurements suggest a light-weighted metallicity above the cutoff of -0.4 in the MIUSCAT-IR models, the optical star formation history carries weight down to $[\mathrm{M} / \mathrm{H}]=-1.71$ at the level of $5-10$ per cent in the centre, to 50 per cent in the very outer parts $(>10 \operatorname{arcsec})$. As mentioned above, this could lead to an underestimation of the population M/L by 10-20 per cent (Meidt et al. 2014), which would make our final IMF lighter, more consistent with Kroupa/Chabrier. We add an additional 20 per cent error on to each $\mathrm{M} / \mathrm{L}_{\text {pop.salp }}$ estimate here to take this systematic into account.

Although the CO emission in this object is well fit by an exponential disc model (as shown in the top left panel of Fig. 2), this source is fairly edge-on and so a central hole in the $\mathrm{CO}$ emitting disc cannot be ruled out. A hole is visible in the distribution of $\mathrm{H} \beta$ flux in our Spectrographic Areal Unit for Research on Optical Nebulae maps, however, we do not know if the distribution of molecular gas has the same feature. If present, such a hole could lead to a spurious decrease of the inner dynamical M/L. An exponential disc model without a hole is preferred by our MCMC modelling. However, higher resolution observations will be required to determine the presence/absence of a central hole entirely.

In addition, if present, hot dust emission from the torus around an AGN in this object could affect the innermost M/L measurement. This object is classified as a broad-line AGN in the SDSS (York et al. 2000), but evidence for nuclear activity was not found in our ATLAS $^{3 \mathrm{D}}$ observations (e.g. Nyland et al. 2016). In any case, given the uncertainty in this innermost measurement, we do not consider it in the rest of this work. Including/removing this point does not alter any of our conclusions.

\subsection{Comparison with previous works}

Given that this is the first time that IMF parameters have been derived using molecular gas, it is important to compare our results to those found by other authors. As our targets are drawn from the ATLAS ${ }^{3 \mathrm{D}}$ survey, they are included in the study of Cappellari et al. (2012) who derive their own IMF mismatch parameter for each source. They assume each source has a single applicable M/L, but given the relatively flat gradients, we see here that it is not unreasonable to compare their value to that found in each of our radial bins. This comparison is shown in Fig. 3. The radial bins within each source are shown in the same colour, as indicated in the legend.

In general, we find good agreement between our radially resolved approach and that unresolved study, with our values differing by $\lesssim 0.1$ dex from those of Cappellari et al. (2012). The exceptions are NGC4429 (where our $\alpha_{\text {dyn }}$ is slightly higher than that of Cappellari et al. 2012) and IC0719, where our $\alpha_{\text {dyn }}$ is radically different. It should be noted, however, that IC0719 was explicitly excluded from

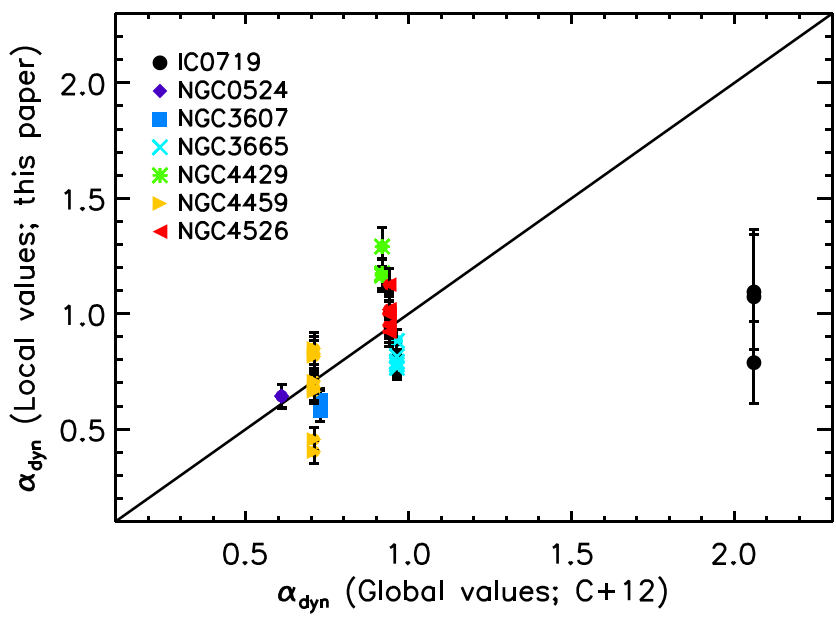

Figure 3. Comparison of the global IMF mismatch factor found by Cappellari et al. (2012) with the local values derived in this work. The radial bins within each source are shown in the same colour and symbol, as indicated in the legend. A one-to-one correlation is shown with the black line to guide the eye.

much of the analysis in Cappellari et al. (2012, 2013) because of the strong gradient in age within the object which makes unresolved analyses (where $\mathrm{M} / \mathrm{L}$ gradients are assumed to be absent) unreliable. Our resolved analysis should be more reliable in such a case.

We hence conclude that overall, our study is in good agreement with the dynamical studies of Cappellari et al. (2012, 2013), as expected, given the analysis conducted in Davis et al. (2013a) comparing $\mathrm{CO}$ and stellar kinematics.

Two of our objects were included in the work of Conroy \& van Dokkum (2012), NGC0524 and NGC4459. That work used gravity sensitive stellar features to spectroscopically derive the IMF normalization and, as such, is interesting to compare with our dynamical method. NGC0524 was found in Conroy \& van Dokkum (2012) to have an IMF normalization similar to the Milky Way $\left(\alpha_{\text {pop,Mw }}\right.$ $=1.08$ ). When transformed to our system with a Salpeter IMF as the reference, this equates to $\alpha_{\text {pop,salp }}=0.7$. Our value averaging over all radii is consistent with this $\left(<\alpha_{\text {dyn }}>=0.66\right)$. NGC4459 in our work has a Kroupa-like IMF normalization $\left(\alpha_{\text {dyn }}=0.70\right)$, while Conroy \& van Dokkum (2012) report a light IMF $\alpha_{\text {pop,salp }}$ of 0.52 ( $\alpha_{\text {pop,Mw }}$ of 0.8 ). This difference is significant on an $\approx 2 \sigma$ level. The disagreement in this object highlights the point made by Smith (2014) that further work must be done in this field to compare techniques. Overall, in this work, however, with only two comparison objects, it is hard to draw general conclusions.

\subsection{The need for IMF variation}

Our objects show that no single IMF can match the observations. For instance, in objects like NGC0524, NGC3607 and NGC4459, the enclosed mass implied, if the stellar population had a Salpeter IMF, is greater than the total dynamical mass. As this would be physically impossible, these objects clearly need a lighter IMF. On the other hand, objects such as NGC3665, NGC4429 and NGC4526 seem to need a heavier IMF to explain their gas kinematics.

\subsection{Variation with galaxy parameters}

In the sections above, we have shown evidence that the IMF varies both within and between massive ETGs, with a range of IMF 
mismatch parameters being found in our sample of seven molecularrich ETGs. In this section, we attempt to determine if the IMF mismatch parameter correlates with any local properties of the galaxy at those locations.

We here utilize the ATLAS ${ }^{3 \mathrm{D}}$ IFU data to derive the average stellar velocity dispersion, circular velocity and stellar populations parameters (age, metallicity, $[\alpha / \mathrm{Fe}]$ ) found in the IFU bins that fall within each beam-width radial region. These radial bins are elliptical (with an axial ratio defined by the galaxy inclination) and are the same as those used to derive the dynamical IMF constraints. The weighted mean of these variables within each annulus is shown as the point, with the error on the weighted mean as horizontal error bar on each point in the following figures. We use these estimates to explore putative correlations between these parameters and the IMF, as suggested by other authors (e.g. Cappellari et al. 2012; Conroy \& van Dokkum 2012; La Barbera et al. 2013; Martín-Navarro et al. 2015b).

We begin by showing the radial variation of each of these key parameters in Fig. 4. Our objects tend to have fairly flat alphaenhancement and velocity dispersion gradients within the region probed, negative metallicity gradients and a dichotomy of age profiles. In the rest of this section, we show how these radial variation compare with the $\alpha_{\text {dyn }}$ values we derive above.

\subsubsection{IMF variation with dynamical properties}

The top panel of Fig. 5 shows the IMF mismatch parameter $\left(\alpha_{\text {dyn }}\right)$ found within each radial bin of our sources, plotted against the mean stellar velocity dispersion within that $\operatorname{bin}^{3}$ (Cappellari et al. 2011). Also shown are the coloured lines that denote the $\alpha_{\text {dyn }}$ parameter of a heavy, Salpeter, Kroupa and Chabrier IMF. No clear and consistent correlation is seen in this figure, either between objects or radially within individual galaxies. A black dot-dashed line is shown denoting the best-fitting correlation of Cappellari et al. (2012), with grey shading showing its scatter.

The bottom panel of Fig. 5 shows the IMF mismatch parameter $\left(\alpha_{\text {dyn }}\right)$ found within each radial bin of our sources, plotted against the circular velocity (measured from our MGE models at the central radius of the bin). The circular velocity gives a measure of the depth of the potential and any systematic variation of the IMF with this parameter could imply that the potential of the host galaxy plays a role in determining the IMF. No clear correlation is seen in this figure, however, either between objects or radially within individual galaxies. This is discussed further in Section 6.

\subsubsection{IMF variation with stellar population parameters}

Fig. 6 shows the IMF mismatch parameter $\left(\alpha_{\text {dyn }}\right)$ found within each radial bin of our sources, plotted against the weighted mean SSP equivalent age, metallicity and alpha-enhancement of the stars within that bin (in the top, centre and bottom panels, respectively). As shown above, labelled coloured lines denote the $\alpha_{\text {dyn }}$ parameter of a heavy, Salpeter, Kroupa and Chabrier IMF.

No clear correlation is observed between $\alpha_{\text {dyn }}$ and these parameters, either locally or globally. These findings are consistent with the weak or absent global correlations reported in McDermid et al. (2014) using the stellar dynamics based $\alpha_{\text {dyn }}$ values from Cappellari et al. (2012). We discuss further all the results on the variation of the IMF with galaxy parameters in Section 6.

\footnotetext{
${ }^{3}$ Available from www.purl.org/atlas3d
}

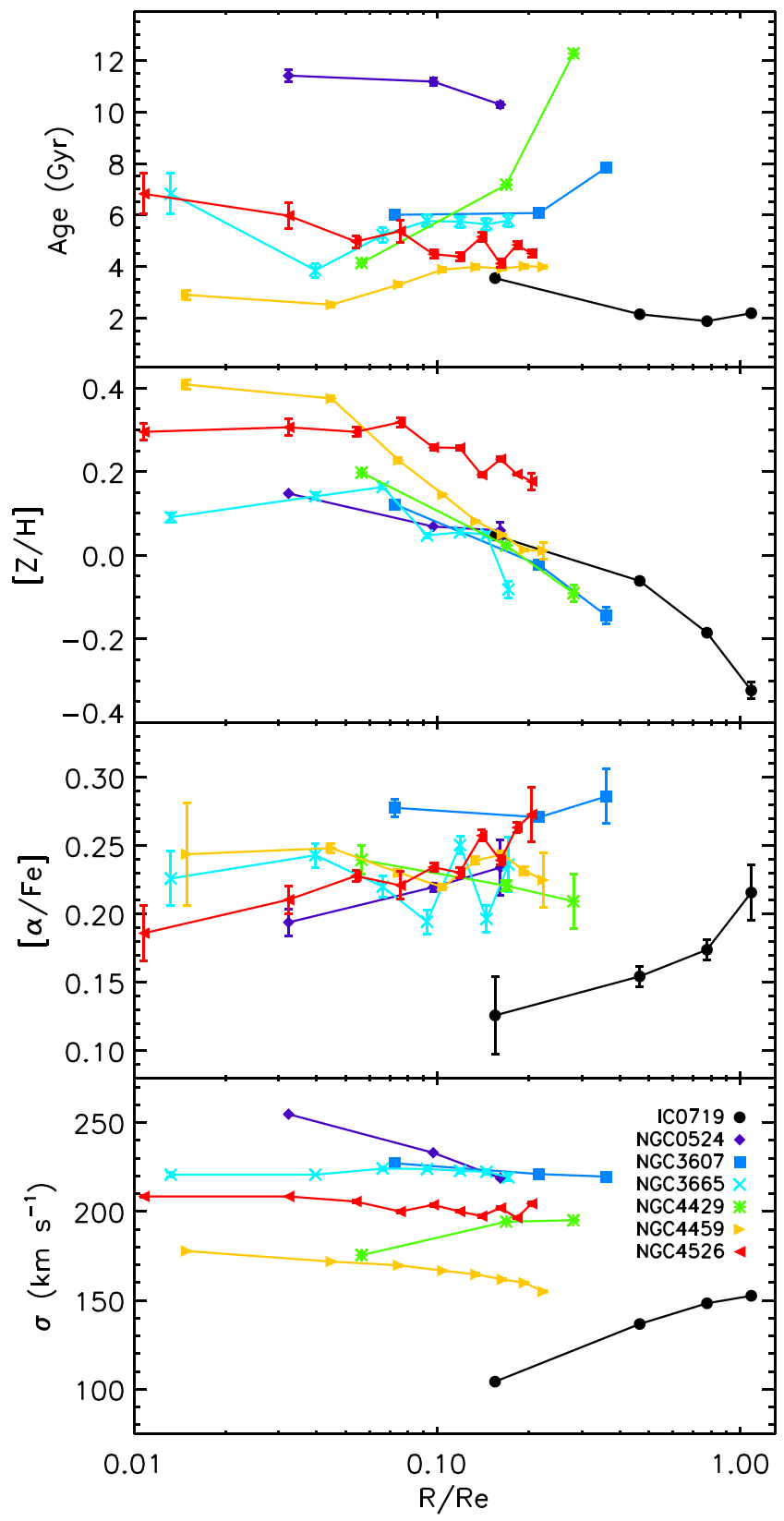

Figure 4. Radial variation of resolved SSP equivalent stellar population parameters measured within our elliptical beam-width bins. We show the weighted mean age, metallicity and alpha-enhancement of the stars, in the first, second and third panel, respectively. Also shown in the fourth panel is the radial variation of the velocity dispersion.

\subsection{Main uncertainties}

In this section, we consider the main uncertainties that could affect the results derived following the method above. These can roughly be split into two subsets: issues with the modelling process and breakdown of the physical assumptions we make to simplify complicated astrophysical problems.

\subsubsection{Dust and its effect on luminous mass models}

In order to convert the velocity profiles estimated from our dynamical modelling to dynamical M/L measurements, as described above, we here parametrize the luminous matter distribution using MGE 

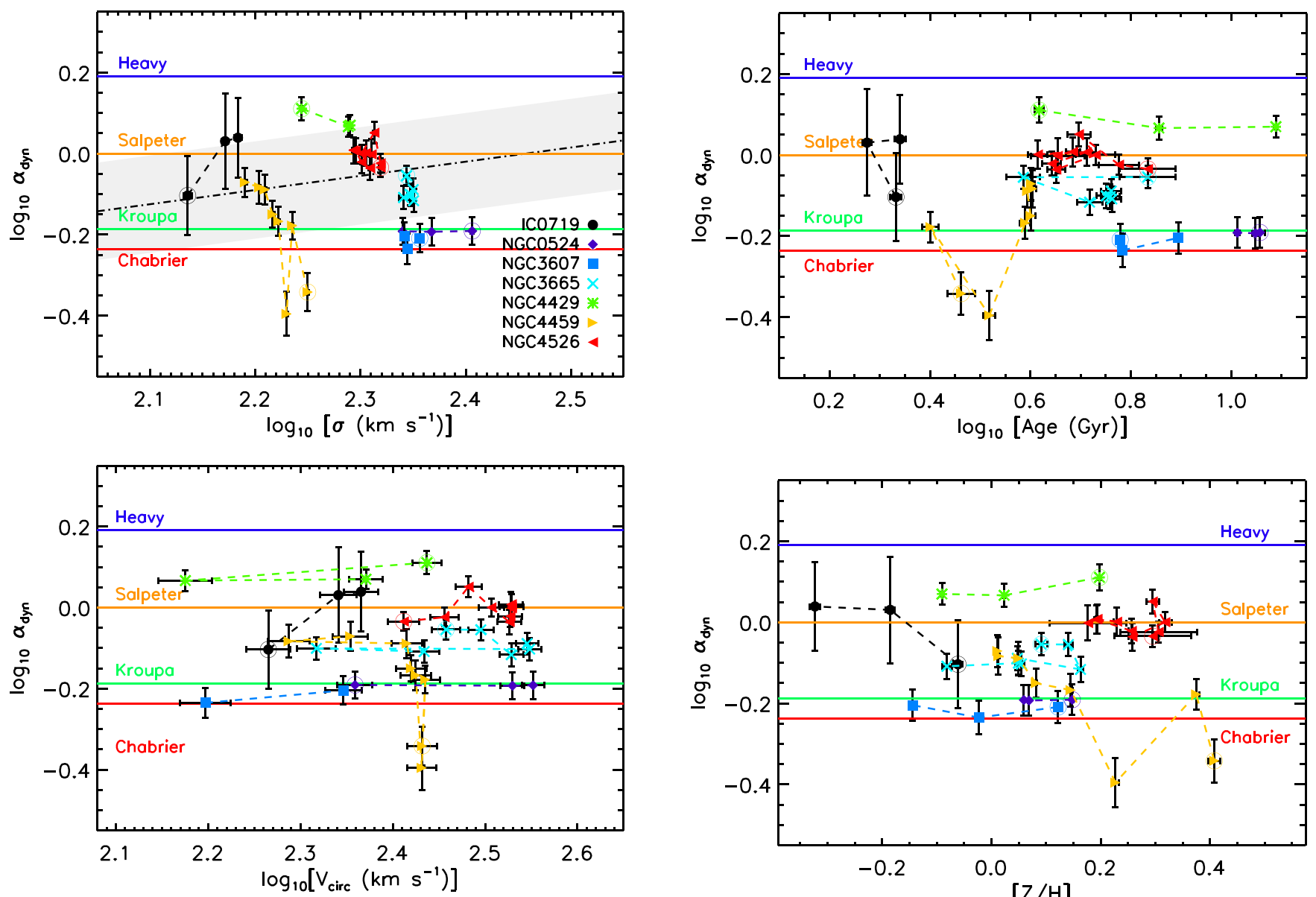

Figure 5. The base-10 logarithm of the IMF mismatch parameter $\left(\alpha_{\mathrm{dyn}}\right)$, plotted against resolved dynamical properties of the host galaxy. Top panel: $\log$ of the mean stellar velocity dispersion within each bin. Bottom panel: the log of the circular velocity (measured at the central radius of the bin). In both plots, individual radial bins within each source are shown in the same colour and symbol, as denoted in the legend. The radial bins are joined with a dashed line of the appropriate colour and the innermost bin we consider is highlighted with a larger open circle in addition to the normal symbol, in order to allow readers to discern radial trends. Also shown are solid coloured lines that denote the $\alpha_{\text {dyn }}$ parameter of a heavy, Salpeter, Kroupa and Chabrier IMF. A black dot-dashed line is shown in the top panel, denoting the best-fitting correlation of Cappellari et al. (2012, 2013) and the grey bar around it denotes the reported scatter around that relation of 0.12 dex.

models of the stellar light distribution. However, it is possible that remaining dust contamination would cause us to underestimate the luminosity of the stars in some parts of our object and thus bias the derived dynamical $\mathrm{M} / \mathrm{L}$. The analysis conducted here is especially affected by this form of uncertainty, as these objects have been selected to host a cold ISM. In order to minimize this issue, our MGE models were constructed from images at the longest wavelength available, in order to minimize dust contamination. The models are also carefully fitted to remove any contamination from dust still visible in the image (via masking of affected regions). Such a process has been shown to work well at recovering the intrinsic light distribution in such systems (Cappellari 2002). Some underlying uncertainty remains which could cause our dynamical M/Ls to be biased to high values. The objects with the most dust obscuration usually have low predicted IMF normalizations (Kroupa/Chabrier
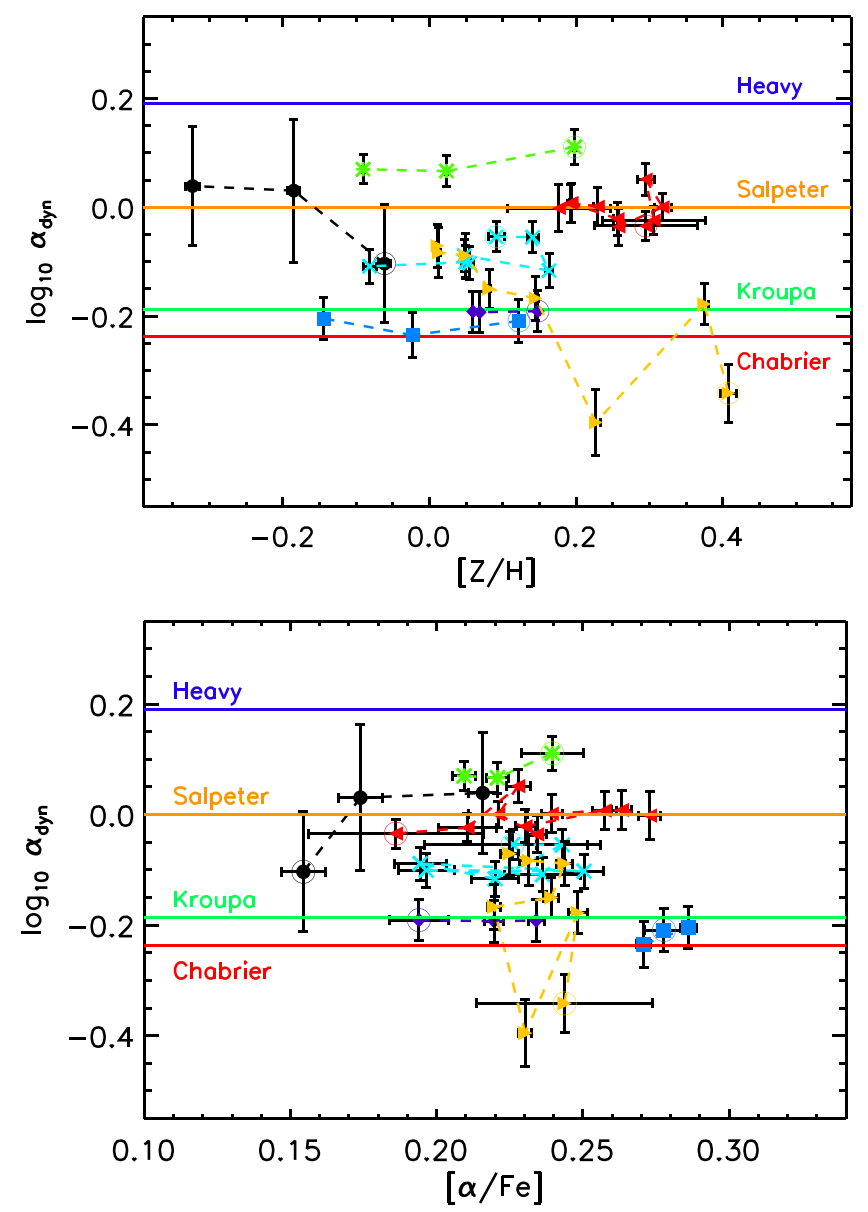

Figure 6. As Fi. 5, but showing the logarithm of the IMF mismatch parameter $\left(\alpha_{\mathrm{dyn}}\right)$, plotted against resolved SSP equivalent stellar population parameters within that bin. These are the mean age, metallicity and alphaenhancement of the stars, in the top, middle and bottom panel, respectively.

like), however, suggesting that this uncertainty cannot remove the need for a heavy IMF in some objects.

\subsubsection{Non-circular motions and gas velocity dispersion}

In this analysis, we assume that the gas is purely in circular rotation. If significant non-circular motions (e.g. inflow, outflow, streaming 
motions) exist within their gas reservoirs, then this could affect our analysis.

Randriamampandry et al. (2015) studied the effect of non-circular motions on the derivation of mass profiles in detail and showed dramatic variations can be caused in strongly barred galaxies if the bar is orientated in specific directions with respect to our line of sight. In order to avoid this problem, we have purposefully selected sample galaxies with regular, relaxed, dynamically cold discs (see Davis et al. 2013a) in galaxies that are not strongly barred (or the gas is within the inner Lindblad resonance of the bar where gas inflowing motions are minimal; Combes \& Gerin 1985; Shlosman, Frank \& Begelman 1989).

Non-circular motions can still be present in objects without bars, for instance, because of other departures from axisymmetry in the gravitational potential (i.e. spiral arms). Such motions are low in amplitude compared to the rotation of these systems (for example, $\approx 10 \mathrm{~km} \mathrm{~s}^{-1}$ in M51, a galaxy with much stronger spiral structure than these early types; Meidt et al. 2013) and add uncertainty at the $\approx 5-10$ per cent level. Such motions also exist only at specific locations within a molecular gas disc and so the smooth gradients found in our objects argue that non-circular motions are not a major effect. The kinematic fits we present here are generally good (with low reduced $\chi^{2}$ values of $1-3$ ), suggesting we do not have significant noncircular motions present within the discs that our models cannot fit.

As discussed above, one object (NGC4459) does show some signatures of non-circular motions. We suspect it has low-level inflowing motions around its innermost ring that are likely the cause of a slightly lower dynamical $\mathrm{M} / \mathrm{L}$ in this region. This difference is small, however, and does not significantly skew the IMF estimate of this source as a whole.

In addition to non-circular motions, an additional source of uncertainty comes from the molecular gas velocity dispersion. A simple prescription for dealing with this is included in our models which allow for a single characteristic velocity dispersion in each disc. Our modelling procedure find values in the range of $5-15 \mathrm{~km} \mathrm{~s}^{-1}$, typical for nearby galaxies (Caldú-Primo et al. 2013). The uncertainty in this measurement has already been marginalized over when producing the error bars on the $\mathrm{M} / \mathrm{L}$ and $\alpha_{\text {dyn }}$ measurements. In reality, however, the velocity dispersion could change with radius and position within the gas disc. If this increase occurs within the flat part of the galaxy rotation curve, away from the central regions of the galaxy, then no bias would be expected in our dynamical estimates as this would simply broaden the linewidth of the gas symmetrically around the same best-fitting value. In the central part of the galaxy where beam smearing is important, however, an increase in velocity dispersion could cause a slight overestimation of $\mathrm{M} / \mathrm{L}_{\text {dyn }}$ (see e.g. Barth et al. 2016).

In order to quantify the size of the effect a variable gas velocity dispersion could have on our derived parameters, we re-ran the modelling process for NGC4459, allowing the velocity dispersion to vary independently in each of the beamwidth-sized radial regions we fit. NGC4459 was chosen for this test, as it is the object in our sample most likely to suffer from these problems (as argued above). While some variation of the velocity dispersion was preferred by the fit (variations of $<5 \mathrm{~km} \mathrm{~s}^{-1}$ ), this had very little effect on the derived $\mathrm{M} / \mathrm{L}$, causing a mean absolute variation in the derived $\mathrm{M} / \mathrm{L}_{\text {dyn }}$ values of less than 3 per cent, which is small compared to the other errors.

\subsubsection{Dark matter and gas mass}

IMF analyses are generally affected by the assumptions they make for the distribution of dark matter. It can be very hard to separate

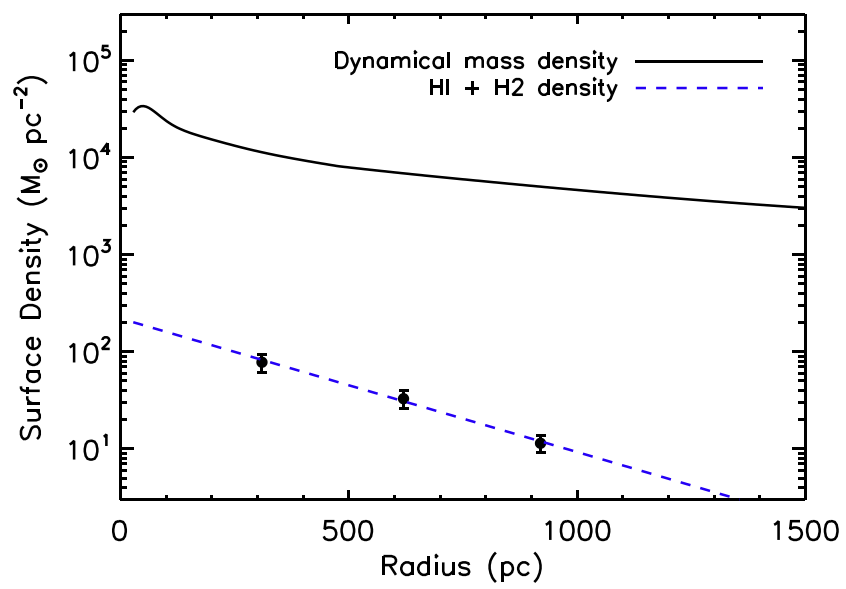

Figure 7. Mass surface densities in NGC0524. The dynamical mass density (black line) is derived from the observed circular velocity curve. The molecular and atomic gas density points are from Martig et al. (2013), while the blue line is the best fitting exponential disc model. The cold gas contributes at most a hundredth of the mass density at any radius.

the effect of extra mass caused by a heavy IMF from that caused by a different dark matter distribution. In this work, we are helped, however, by only being able to probe the central regions of ETGs. Various studies of the dark matter content in ETGs have showed that these inner regions are totally dominated by the mass of stars. For instance, Cappellari et al. (2013) find typical dark matter fractions of 13 per cent within the effective radius of ETGs. As our observations typically probe only within a quarter of the effective radius, we expect the dark matter contribution to the mass profile to be very low.

In addition to dark matter, the mass of any ISM material present also contributes to the total dynamical mass of the system. The hot phases of the ISM are diffuse enough to not contribute significantly to the mass budget in the central parts of galaxies, but both $\mathrm{HI}$ and $\mathrm{H}_{2}$ masses could potentially affect the mass profiles we derive. This has been shown to be an important affect in gas-rich objects, especially at high redshifts (e.g. De Breuck et al. 2014). It should be possible to include the gas mass in our calculations and thus take it into account. However, given the uncertainty in $X_{\mathrm{CO}}$ (the conversion between $\mathrm{CO}$ luminosity and mass), this could induce large errors. In the centre of ETGs, however, the density of the ISM material is insignificant compared to the stellar mass density (for any sensible $X_{\mathrm{CO}}$ ). This is shown graphically in Fig. 7 which shows the dynamical mass surface density and the cold gas surface density in NGC0524 which was previous studied in detail by Crocker et al. (2011) and Martig et al. (2013). The dynamical mass surface density (assuming the mass is spherically distributed) is derived from the rotation curve as

$\Sigma_{\mathrm{dyn}}=\frac{V^{2}}{\pi G r}$,

where $V$ is the circular velocity derived from our MGE and M/L model fitting, $G$ is the gravitational constant and $r$ is the radius. This is compared to the atomic and molecular gas surface density (calculated assuming the mass is distributed in a flat disc) which is taken from the observations of Martig et al. (2013; in this object, the molecular gas density dominates over the atomic gas). The cold gas contributes at most 1 per cent of the mass density at any radius, making it dynamically unimportant. We thus consider it unlikely that neglecting the mass of gas in our fitting procedure biases our results. 


\section{DISCUSSION}

In the sections above, we presented our method to measure dynamical $\mathrm{M} / \mathrm{L}$ as a function of radius in seven ETGs. We compared these measurements to stellar population parameters derived from IFU data in order to estimate the IMF mismatch parameter and how it varies with radius. In this section, we discuss these results and what they may mean in a wider context.

\subsection{Radial M/L variation in molecular gas-rich ETGs}

In the right-hand panel of Fig. 2, we show the M/L gradients present within our seven target ETGs. To first order, ETGs such as these have uniformly old stellar populations with a frosting of new stars formed in the most recent gas-rich merger/accretion episode (see e.g. Young et al. 2014). These young stars have a very low mass-fraction, but may contribute significant amounts of light, especially in the blue/UV. This is likely to lower the M/L of the stellar population within the molecular gas disc.

In a disc of star-forming gas, the new stars will be formed following the spatial distribution of the gas. Unless the galaxy light also follows an exponential disc profile, we thus expect mass-to-light gradients to exist within the disc. Several of our objects do show small $\mathrm{M} / \mathrm{L}$ gradients (both in $\mathrm{M} / \mathrm{L}_{\text {pop }}$ and $\mathrm{M} / \mathrm{L}_{\mathrm{CO}}$ ) and several other objects have smaller variations at individual radii, which relate to specific features in their gas distribution (see the discussion of individual sources above). Overall, however, our analysis shows that $\mathrm{M} / \mathrm{L}$ gradients do not seem to be very strong in the inner parts of these objects (whichever method you use to derive them). This is likely because of the low star formation rates of these sources. The objects that do seem to have some $\mathrm{M} / \mathrm{L}$ variations are also those with the highest specific star formation rates (Davis et al. 2014). In the other objects, even luminous $\mathrm{O} / \mathrm{B}$ stars cannot outshine the more numerous old stars in the cores of these objects (especially in the long wavelength bands we use to create our luminous mass models).

\subsection{IMF variation and correlations with galaxy parameters}

Above, we showed that no single IMF can match the observations of gas kinematics in our sample ETGs and thus that the IMF appears to be variable. With this technique, we are only sensitive to the total IMF normalization and are unable to determine if the extra mass comes from high- or low-mass stars. Other analyses suggest the latter to be more likely but we cannot comment further on this here.

In Section 5.4, we showed that the IMF normalization derived in radial bins in our sample ETGs does not seem to correlate with various galaxy parameters. This is true both locally within individual galaxies and globally between systems. This is true for both dynamical (galaxy circular velocity or stellar velocity dispersion; see Fig. 5) and stellar population parameters (age, metallicity and alpha-enhancement).

The galaxy parameters we consider here all show significant radial variation in galaxies. This highlights the importance of understanding aperture effects which complicate the comparison of the IMF derived from (spatially weighted) kinematics with luminosityweighted stellar populations analyses. This study allows us to remove potential issues with aperture effects that were faced by previous dynamical studies, by considering the local variations in both IMF and stellar populations. We still find no evidence for a correlation between IMF and stellar populations consistent with the weak or absent correlations reported in McDermid et al. (2014).
The lack of correlation with stellar velocity dispersion may seem surprising when one remembers the good agreement between the IMF parameters derived in this study and those from Cappellari et al. (2012; as shown in Section 5.2), who report a strong correlation between these variables. The best fit reported by Cappellari et al. (2012) is shown in Fig. 5. Our objects do not seem to individually follow this relation, but as a whole, the majority of the points fall within $\approx 0.2 \mathrm{dex}$ of the correlation. The $1 \sigma$ scatter around the best fit in Cappellari et al. (2012) was 0.12 dex (shown as the grey shaded region in Fig. 5), and as $\approx 2 / 3$ of our points fall within this region, we cannot rule out the possibility that our objects' global normalizations have been drawn from this underlying distribution. If this is the case, however, then any correlation with stellar velocity dispersion appears to be global, rather than also appearing locally within galaxies.

In this work, we find no significant correlations with any of the studied dynamical or population parameters and thus it seems that substantial disagreements remain between different studies of IMF variation. For instance, the resolved stellar population study of Martín-Navarro et al. (2015b) found a strong correlation between the IMF normalization and metallicity and the work of Conroy \& van Dokkum (2012) suggested that the strongest correlation was between the IMF and alpha-element enhancement. Our analysis does seem to yield reasonably consistent $\alpha_{\text {dyn }}$ values to those found in other works (e.g ATLAS ${ }^{3 \mathrm{D}}$; see Fig. 3), however, small differences are found in one of the two objects that overlap with the study of Conroy \& van Dokkum (2012). Future studies of a greater number of objects that will help reveal if these studies still lack internal consistency (Smith 2014). Given that our results are consistent with those using stellar kinematics, however, it does suggest that if a problem exists in current analyses, it is likely to lie in the uncertainties inherent in stellar population modelling, rather than our understanding of galaxy dynamics.

\section{CONCLUSIONS}

In this paper, we present the first spatially resolved study of the IMF in external galaxies derived using a dynamical tracer of the M/L. We do this using molecular gas kinematics in seven ETGs selected from the ATLAS ${ }^{3 \mathrm{D}}$ survey. We compare these measurements to stellar population parameters derived from star formation histories in order to estimate the IMF mismatch parameter and shed light on the variation of the IMF within ETGs.

We find that the $\mathrm{M} / \mathrm{L}$ gradients in the inner parts of our target objects are not very strong (independent of the method used to derive them). Several objects do show modest $\mathrm{M} / \mathrm{L}_{\mathrm{CO}}$ gradients, while other have smaller $\mathrm{M} / \mathrm{L}_{\mathrm{CO}}$ variations at individual radii which relate to specific features in their gas distribution. The objects that do seem to show $\mathrm{M} / \mathrm{L}_{\mathrm{CO}}$ variations are also those with the highest specific star formation rates. The majority of these slight gradients are also present in the stellar population analyses, but not all are.

We confirm that the IMF appears to vary when comparing different massive ETGs. Some of our target objects require a light IMF, otherwise their stellar population masses would be greater than their dynamical masses. In contrast, other systems seem to require heavier IMFs to explain their gas kinematics. We find good agreement between our IMF normalizations derived using molecular gas kinematics and those derived by ATLAS ${ }^{3 \mathrm{D}}$ using stellar kinematics. This provides an independent check on the stellar kinematic results, suggesting that if a problem exists in current analyses, it is more likely to lie in the stellar population modelling, rather than in the dynamics. We note that this agreement occurs despite our objects being 
the hardest to model using stellar methods (due to their strong discs of gas and dust). Thus, with this technique, we can, in principle, extend studies of the IMF normalization to more gas-rich systems.

We do not see strong variation of the IMF normalization with galaxy dynamical or stellar population properties in this work, either locally or globally. This study allows us to remove potential biases due to aperture effects that were faced by previous studies. By considering the local variations in both IMF and stellar populations, we find no evidence for a correlation between IMF and stellar populations, consistent with the weak or absent correlations reported in McDermid et al. (2014). In this work alone, we also do not find a convincing connection between galaxy dynamics and the IMF. Future works of this type will be required to show if the lack of observed correlations is real or due to low number statistics.

In the future, larger studies of molecular gas kinematics can help to disentangle the cause of IMF variation. This method provides an independent check on the conclusions of other dynamical methods and, in addition, can provide dynamical information on IMF gradients within individual galaxies. Projects such as the CARMAEDGE survey (Utomo et al. 2015a) and the MASSIVE survey (Ma et al. 2014), which combine IFU kinematics with resolved molecular gas observations, should allow extension of this technique to large samples of galaxies across the Hubble sequence.

\section{ACKNOWLEDGEMENTS}

TAD acknowledges support from a Science and Technology Facilities Council Ernest Rutherford Fellowship and thanks F. van de Voort for fruitful discussions.

This paper is based on observations carried out with the IRAM Thirty Meter Telescope, the IRAM Plateau de Bure interferometer and the CARMA interferometer. IRAM is supported by INSU/CNRS (France), MPG (Germany) and IGN (Spain). Support for CARMA construction was derived from the states of California, Illinois, and Maryland, the James S. McDonnell Foundation, the Gordon and Betty Moore Foundation, the Kenneth T. and Eileen L. Norris Foundation, the University of Chicago, the Associates of the California Institute of Technology, and the National Science Foundation.

This paper is based in part on observations made with the NASA/ESA HST, and obtained from the Hubble Legacy Archive, which is a collaboration between the Space Telescope Science Institute (STScI/NASA), the Space Telescope European Coordinating Facility (ST-ECF/ESA) and the Canadian Astronomy Data Centre (CADC/NRC/CSA). This research has made use of the NASA/IPAC Extragalactic Database (NED) which is operated by the Jet Propulsion Laboratory, California Institute of Technology, under contract with the National Aeronautics and Space Administration.

\section{REFERENCES}

Alatalo K. et al., 2013, MNRAS, 432, 1796

Auger M. W., Treu T., Gavazzi R., Bolton A. S., Koopmans L. V. E., Marshall P. J., 2010, ApJ, 721, L163

Barth A. J., Darling J., Baker A. J., Boizelle B. D., Buote D. A., Ho L. C., Walsh J. L., 2016, ApJ, 823, 51

Bastian N., Covey K. R., Meyer M. R., 2010, ARA\&A, 48, 339

Caldú-Primo A., Schruba A., Walter F., Leroy A., Sandstrom K., de Blok W. J. G., Ianjamasimanana R., Mogotsi K. M., 2013, AJ, 146, 150

Cappellari M., 2002, MNRAS, 333, 400

Cappellari M., Copin Y., 2003, MNRAS, 342, 345

Cappellari M., Emsellem E., 2004, PASP, 116, 138

Cappellari M. et al., 2011, MNRAS, 413, 813
Cappellari M. et al., 2012, Nature, 484, 485

Cappellari M. et al., 2013, MNRAS, 432, 1709

Cenarro A. J., Gorgas J., Vazdekis A., Cardiel N., Peletier R. F., 2003, MNRAS, 339, L12

Chabrier G., 2003, PASP, 115, 763

Clauwens B., Schaye J., Franx M., 2016, MNRAS, 462, 2832

Combes F., Gerin M., 1985, A\&A, 150, 327

Combes F., Young L. M., Bureau M., 2007, MNRAS, 377, 1795

Conroy C., van Dokkum P. G., 2012, ApJ, 760, 71

Crocker A. F., Bureau M., Young L. M., Combes F., 2011, MNRAS, 410, 1197

Davis T. A., 2014, MNRAS, 443, 911

Davis T. A. et al., 2011a, MNRAS, 414, 968

Davis T. A. et al., 2011b, MNRAS, 417, 882

Davis T. A. et al., 2013a, MNRAS, 429, 534

Davis T. A., Bureau M., Cappellari M., Sarzi M., Blitz L., 2013b, Nature, 494, 328

Davis T. A. et al., 2014, MNRAS, 444, 3427

De Breuck C. et al., 2014, A\&A, 565, A59

Dutton A. A., Macciò A. V., Mendel J. T., Simard L., 2013, MNRAS, 432, 2496

Emsellem E., Monnet G., Bacon R., 1994, A\&A, 285, 723

Ferreras I., La Barbera F., de la Rosa I. G., Vazdekis A., de Carvalho R. R., Falcon-Barroso J., Ricciardelli E., 2013, MNRAS, 429, L15

Krajnovic D., McDermid R. M., Cappellari M., Davies R. L., 2009, MNRAS, 399, 1839

Kroupa P., 2001, MNRAS, 322, 231

Kroupa P., 2002, Science, 295, 82

La Barbera F., Ferreras I., Vazdekis A., de la Rosa I. G., de Carvalho R. R., Trevisan M., Falcon-Barroso J., Ricciardelli E., 2013, MNRAS, 433, 3017

La Barbera F., Vazdekis A., Ferreras I., Pasquali A., Cappellari M., Martín-Navarro I., Schönebeck F., Falcón-Barroso J., 2016, MNRAS, 457, 1468

McDermid R. M. et al., 2014, ApJ, 792, L37

McDermid R. M. et al., 2015, MNRAS, 448, 3484

Ma C.-P., Greene J. E., McConnell N., Janish R., Blakeslee J. P., Thomas J., Murphy J. D., 2014, ApJ, 795, 158

Martig M. et al., 2013, MNRAS, 432, 1914

Martín-Navarro I., Barbera F. L., Vazdekis A., Falcon-Barroso J., Ferreras I., 2015a, MNRAS, 447, 1033

Martín-Navarro I. et al., 2015b, ApJ, 806, L31

Meidt S. E. et al., 2012, ApJ, 744, 17

Meidt S. E. et al., 2013, ApJ, 779, 45

Meidt S. E. et al., 2014, ApJ, 788, 144

Nyland K. et al., 2016, MNRAS, 458, 2221

Parma P., de Ruiter H. R., Fanti C., Fanti R., 1986, A\&AS, 64, 135

Posacki S., Cappellari M., Treu T., Pellegrini S., Ciotti L., 2015, MNRAS, 446, 493

Randriamampandry T. H., Combes F., Carignan C., Deg N., 2015, MNRAS, 454, 3743

Röck B., Vazdekis A., Peletier R. F., Knapen J. H., Falcón-Barroso J., 2015, MNRAS, 449, 2853

Salpeter E. E., 1955, ApJ, 121, 161

Sault R. J., Teuben P. J., Wright M. C. H., 1995, in Shaw R. A., Payne H. E., Hayes J. J. E., eds, ASP Conf. Ser. Vol. 77, Astronomical Data Analysis Software and Systems IV. Astron. Soc. Pac., San Francisco, p. 433

Schiavon R. P., 2007, ApJS, 171, 146

Sheth K. et al., 2010, PASP, 122, 1397

Shlosman I., Frank J., Begelman M. C., 1989, Nature, 338, 45

Smith R. J., 2014, MNRAS, 443, L69

Tortora C., Romanowsky A. J., Napolitano N. R., 2013, ApJ, 765, 8

Tortora C., La Barbera F., Napolitano N. R., 2016, MNRAS, 455, 308

Treu T., Auger M. W., Koopmans L. V. E., Gavazzi R., Marshall P. J., Bolton A. S., 2010, ApJ, 709, 1195

Utomo D., Blitz L., Bolatto A., Wong T., Vogel S., 2015a, IAU General Assembly, 22, 2257914 
Utomo D., Blitz L., Davis T., Rosolowsky E., Bureau M., Cappellari M., Sarzi M., 2015b, ApJ, 803, 16

van Dokkum P. G., Conroy C., 2010, Nature, 468, 940

Vazdekis A., Casuso E., Peletier R. F., Beckman J. E., 1996, ApJS, 106, 307

Vazdekis A., Ricciardelli E., Cenarro A. J., Rivero-González J. G., DíazGarcía L. A., Falcón-Barroso J., 2012, MNRAS, 424, 157

York D. G. et al., 2000, AJ, 120, 1579
Young L. M., Bureau M., Cappellari M., 2008, ApJ, 676, 317

Young L. M. et al., 2014, MNRAS, 444, 3408

Zieleniewski S., Houghton R. C. W., Thatte N., Davies R. L., 2015, MNRAS, 452,597

This paper has been typeset from a $\mathrm{T}_{\mathrm{E}} \mathrm{X} / \mathrm{L} \mathrm{T} \mathrm{E} \mathrm{X}$ file prepared by the author. 